Ann. Sci. forest., 1979, 36 (4), 331-339.

\title{
Premières données sur la nématofaune associée aux dépérissements du pin maritime dans l'Ouest de la France
}

\author{
P. BAUJARD, A. BOULBRIA *, R. HAM** \\ C. LAUMOND *** ef C. SCOTTO LA MASSESE *** \\ avec la collaboration technique de MINOT J. C. *** et VOISIN R. *** \\ Laboratoire de Botanique, \\ Université de Bordeaux 1 \\ 33405 Talence \\ * Laboratoire de sylviculture ef d'écologie de la pinède landaise, I.N.R.A., \\ 33610 Cestas Principal \\ ** Station de Recherches de Zoologie et de Biocoénotique forestières, I.N.R.A., \\ Ardon, 45160 Olivet \\ *** Station de Recherches sur les Nématodes, I.N.R.A., \\ Centre de Recherches d'Antibes \\ 06602 Antibes
}

\section{Résumé} todes.

Des mortalités inexpliquées de Pinus pinaster nous ont amenés à rechercher la présence de néma-

L'analyse nématologique a révélé une nématofaune importante (32 espèces) dans laquelle nous avons retrouvé Bursaphelenchus lignicolus, dont la pathogénicité sur les pins a déjà été étudiée et démontrée au Japon. L'étude histopathologique révèle la présence de ces nématodes dans les canaux résinifères et les trachéides du bois.

La prospection faite a permis de mettre en évidence une présence de Sirex importante et l'existence, sur arbres sur pied, de champignons de bleuissement.

Le Pin maritime couvre l'essentiel du massif forestier landais sur plus d'un million d'hectares (Gironde, Landes, Lot-et-Garonne) et constitue par ailleurs, des boisements plus ou moins importants dans l'Ouest de la France (Dordogne, Charentes, Vendée, Sarthe, Cher, Morbihan). Bien que cette essence n'y souffre pas de dépérissements aussi catastrophiques que ceux qu'elle vient de subir, du fait de Matsucoccus feytaudi Duc. dans le Sud-Est (Var, Alpes-Maritimes), il est cependant évident que des foyers de mortalité se présentent plus ou moins régulièrement dans le temps et dans l'espace dans les régions du Sud-Ouest et de l'Ouest.

Jusqu'à présent, les forestiers cherchaient l'explication dans une cause «classique », c'est-à-dire attaque d'insectes ravageurs primaires (Thaumetopœa pityocampa Schiff., Clavigesta sylvestrana Curtis) « pourridiés », sècheresse, mauvaise adaptation 
des plants issus de graines exogènes... A vrai dire, à l'exception des ravageurs primaires cités, seule l'Armillaire (Armillariella mellea Karst.) un des trois agents des «pourridiés», pouvait être reconnue de façon indubitable grâce à son mycélium, les deux autres champignons (Fomes annosus (Fr.) Cke. et Rhizina inflata (Schäff)Karst.) n'étant décelables que par leur extériorisation, assez rare, sous forme de carpophores.

Il est difficile de connaître avec précision l'influence réelle de certaines causes de dépérissement telle que la sécheresse. Il est simplement possible de supputer l'action éventuelle d'un déficit hygrométrique momentané par les caractéristiques du biotope, mais toujours a posteriori.

Par ailleurs la sensibilité au froid et l'inadaptation des pins provenant de graines d’origine extérieure (Portugal, Espagne) ont été parfaitement démontrées (Bouvarel, 1960, Illy, 1966).

Devant l'impossibilité d'expliquer un certain nombre de phénomènes de mortalité, deux des auteurs (A. B. et C. SLM.) ont été amenés à se préoccuper, depuis 1972, de l'action possible d'agents phytopathogènes appartenant à un groupe zoologique par ailleurs bien connu pour son action néfaste sur les cultures : les nématodes.

D'abord centrées sur la nématofaune tellurique, ces études ont abouti à établir, à partir d'un millier d'échantillons, une cartographie nématologique du Pin maritime (Scotto La Massèse et Boulbria, 1974 ; Baujard, 1975 ; Baujard et al. à paraître) qui a montré des corrélations beaucoup plus étroites entre les nématodes phytophages et les plantes des strates herbacées et arbustives du couvert végétal qu'avec le Pin maritime.

La publication de travaux japonais (Kiyohara et Tokushiga, 1971 ; Mamiya, $1972 a$ et $b$; Mamiya et Kiyohara, 1972), qui ont mis en évidence la nocuité du nématode Bursaphelenchus lignicolus Mamiya et Kiyohara, 1972 dans la destruction de Pinus densiflora Sieb. et Zucc. et Pinus thumbergii Parl. (400000 à plus de $1000000 \mathrm{~m}^{3}$ de pertes annuelles), nous a incités à rechercher si les troncs de pin n'hébergeaient pas de nématodes susceptibles de jouer un rôle dans les dépérissements. En 1976, nous parvenions à extraire, à partir de sections de troncs d'une quarantaine d'années, prélevées à Lencouacq (Landes), un nombre important de nématodes appartenant à neuf espèces différentes. Depuis lors, les altérations de croissance ont fait l'objet d'une étude nématologique systématique qui a révélé des populations considérables et très variées, appartenant pour la plupart à des espèces jamais encore trouvées dans les analyses de sol.

La présence de nématodes dans le tronc des pins a été notée pour la première fois par Steiner (1934) dans Pinus palustris Mill. et Pinus echinata Mill. Le nématode impliqué fut décrit sous le nom d'Aphelenchoides xylophilus Steiner, 1934, mais il a été rapporté depuis au genre Bursaphelenchus par Nickle (1970). Découvert dans les galeries de Scolytes et dans des zones envahies par des cryptogames dont Ceratostomella pini Bakshi, il est véhiculé par des Scolytes et considéré comme mycophage.

Alors que la présence de nématodes dans les galeries d'insectes xylophages est fréquemment admise (Rühm, 1956), il a fallu attendre les travaux des auteurs japonais ci-dessus évoqués pour qu'un nématode du tronc puisse être rendu responsable, sans équivoque, du dépérissement des conifères. Mamiya et ses collaborateurs ont en effet pu mettre en évidence, par l'histologie, la présence de $B$. lignicolus dans des canaux résinifères du bois très éloignés des galeries des xylophages. De plus l'inoculation de ce nématode à des arbres sains a permis de reproduire les symptômes observés dans les foyers de dépérissement. Une étude récente de Bedding (1972) en Australie montre 
également que certaines espèces de nématodes appartenant au genre Deladenus migrent à partir des galeries de Sirex dans les trachéides et les canaux résinifères de Pinus radiata $\mathrm{D}$. Don où se développent alors des champignons disséminés par l'insecte.

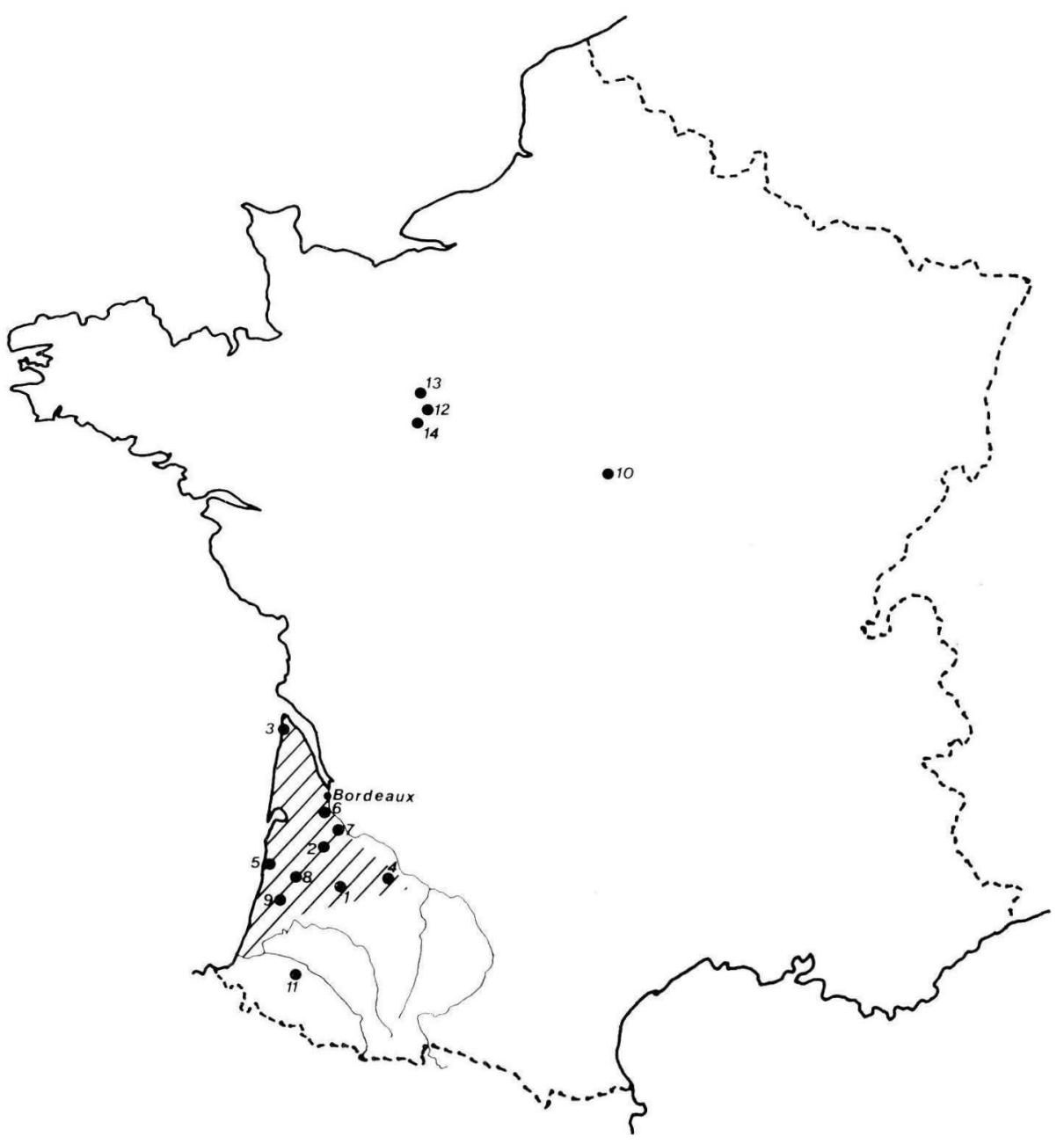

- lieu de prelevement.

massif forestier landais.

FIG. 1. - Répartition géographique des foyers de mortalité.

Geographical distribution of decay foci. 


\section{Foyers de dépérissements (fig. 1)}

Différents types de dépérissements associés à la présence de nématodes dans les troncs ont été recensés :

1. - Mortalité en taches affectant une surface variable comportant de vingt à quatre-vingts arbres morts. C'est l'aspect classique de la «maladie du rond », terme landais désignant une attaque d'Armillaire. Mais la différence fondamentale réside dans le fait que la décadence des arbres atteints est ici particulièrement brutale (un mois en août-septembre) et se produit de façon simultanée pour tous les sujets, ce qui n'est pas le cas de la « maladie du rond » qui évolue lentement.

Dans les deux cas connus : Lencouacq, Belhade (Landes), il s'agit d'arbres adultes de 40 à 50 ans.

2. - Mortalité d'arbres isolés ou par petits bouquets de 2 ou 3. C'est le cas de Naujac (Gironde), sur pins de 40 ans, Saint-Julien-en-Born (Landes) sur pins de 10 ans, les Tureaux (Cher) sur pins de 30 ans, le Narais (Sarthe) sur pins de 10 ans, Parignél'Evêque (Sarthe) et Saint-Mars-la-Brière (Sarthe) sur pins de 50 ans.

3. - Mortalité très diffuse avec des arbres mourant isolément. C'est le cas de Leon (Landes) où seule la moitié supérieure de la cime est atteinte et sèche complètement. Il faut noter que cet aspect peut aussi provenir d'une attaque importante de Dioryctria sylvestrella (plusieurs chenilles sur une longueur de tronc réduite) et, mais de façon moins nette, par plusieurs défoliations successives de Thaumetopoea pityocampa Schiff. surtout sur les jeunes arbres. Dans le cas cité aucun de ces deux ravageurs n'a été décelé.

4. - Symptômes localisés n'entraînant pas obligatoirement la mort de l'arbre mais d'une ou plusieurs branches : le Puch (Gironde) et Uzeste (Landes). Ils s'observent sur de jeunes arbres parfaitement venant de 5 à 7 ans.

5. - Peuplements sans mortalité actuelle, mais présentant un aspect particulièrement souffreteux : Escource (Landes) rappelant l'allure des «pins portugais » dans les années 1962-1965.

Il faut également signaler que nous avons retrouvé des nématodes dans des Pinus laricio dépérissants dans la Sarthe et à Navarrenx (Pyrénées atlantiques). Par ailleurs, dans plus de 80 p. 100 des échantillons observés, les sections de tronc révèlent la présence de cryptogames qui provoquent le bleuissement du bois.

\section{Nématofaune associée aux dépérissements (tabl. 1)}

II convient tout d'abord de noter qu'aucun nématode n'a été décelé dans le bois d'arbres sains ou ne présentant aucun des symptômes ci-dessus décrits en particulier dans le cas de mortalités dues à l'Armillaire (Landes et Forêt de Fontainebleau). 


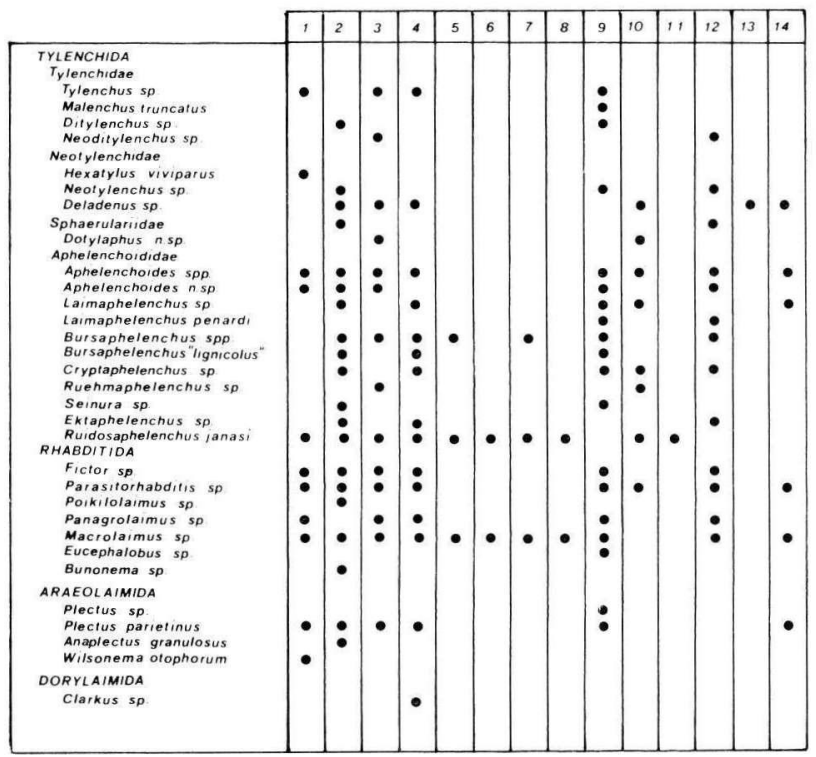

TABLEAU + Repartition geographique de la nématotaune.

La nématofaune épigée recensée sur le Pin maritime se caractérise par sa densité, son homogénéité dans l'espace et sa spécificité par rapport aux populations telluriques. Les plus fortes concentrations ont été recensées à Leon (Landes où nous avons extrait (par la méthode de Baermann) plus de 300000 nématodes pour $732 \mathrm{~g}$ de bois sec (séchage à l'étuve à $105^{\circ} \mathrm{C}$ en fin d'analyse).

Actuellement plus d'une trentaine de genres ont été identifiés appartenant pour la plupart aux Tylenchida et aux Rhabditida. Par ailleurs, quelques espèces appartenant aux genres Tylenchus, Malenchus, Ditylenchus, Bunonema, Plectus, Anaplectus et Wilsonema représentés assez communément dans les sols landais, ont été répertoriées.

En dehors de $B$. lignicolus les autres espèces et genres cités dans tableau 1 sont pou $r$ la plupart considérés comme étant associés aux insectes et incapables de nuire aux végétaux qui les hébergent, mais leur présence en nombre important dans les sections de tronc non infestées par des xylophages remet en cause cette opinion. Des études particulières s'avèrent donc nécessaires pour préciser la biologie et le rôle de ces nématodes dans les dépérissements.

La répartition géographique de cette nématofaune permet de distinguer plusieurs ensembles :

$1^{0}$ Dans six sites échantillonnés (Campet, Les Tureaux, Belhade, Naujac, Parignél'Evêque, Saint-Mars-la-Brière), nous trouvons une population importante de Deladenus sp. dont l'identité n'est pas parfaitement définie. Sur le site des Tureaux, la capture de Sirex adultes prêts à émerger d'un pin encore vert, nous a permis de trouver, dans la cavité cœlomique de l'insecte, le stade parasite (forme Sphaerulariide) du Deladenus sp. alors que le stade mycétophage (forme Neotylenchide) est présent dans le bois (Bedding, 1968). Il faut remarquer que, sur le site de Parigné-l'Evêque, certains arbres échantillonnés ne sont pas parasités par le Sirex cependant présent dans la pinède ; la présence de la forme mycétophage du Deladenus sp. laisse supposer que le Sirex a tenté de pondre sur cet arbre ou bien que les œufs ne se sont pas développés. 
$2^{\circ}$ Dans 3 sites (Campet, Belhade, Leon) on recense Bursaphelenchus lignicolus, qui se distingue des autres espèces du genre par un repli de la lèvre vulvaire et la forme des spicules (Mamiya et Kiyohara, 1972).

La souche landaise possède bien ces deux caractères; de plus, les mensurations et rapports morphométriques ne diffèrent pas significativement de ceux de la souche japonaise (cf. tabl. 2). Toutefois, la forme quadrangulaire de la bursa de l'espèce landaise diffère de celle (cordiforme) figurant dans la description de B. lignicolus; mais les examens effectués sur des exemplaires de $B$. lignicolus reçus du Japon révèlent qu'elle est bien quadrangulaire et non cordiforme comme l'indiquent les illustrations des auteurs.

$3^{0}$ Dans la moitié des sites (tabl. 1), trois autres espèces nouvelles de Bursaphelenchus, apparaissent souvent en grand nombre ; leur rôle doit être précisé.

II n'apparaît pas de corrélations entre les différents types de dépérissement ou mortalité cités et la structure spécifique de la nématofaune recensée. II faut cependant noter sa très grande homogénéité. II ne semble pas en effet y avoir d'espèce liée au milieu géographique, en dehors peut-être de la présence dans le Sud-Est de Ruidosaphelenchus janasi Laumond et Carle, 1971, où il est signalé comme phorétique de Blastophagus destruens Woll., Scolyte inconnu dans les Landes.

\section{TABLEAU 2}

Comparaison des rapports morphométriques et des mensurations

des souches japonaise et landaise de Bursaphelenchus lignicolus

\begin{tabular}{|c|c|c|c|c|c|c|c|c|}
\hline & \multicolumn{4}{|c|}{ ESPECE JAPONAISE } & \multicolumn{4}{|c|}{ ESPECE LANDAISE } \\
\hline & + & $q_{n=40}$ & & $\sigma_{n=30}^{\infty}$ & + & $q_{n=12}$ & + & $\sigma_{n=12}^{\star}$ \\
\hline$L(\mu m)$ & 810 & $710-1010$ & 730 & $590-820$ & 753. & $648-895$ & 692 & $602-750$ \\
\hline a & 40 & $33-46$ & 42,3 & $36-47$ & 43,1 & $37 \cdot 53$ & 42,2 & $37,2-44,6$ \\
\hline$b$ & 10.3 & $9,4-12.8$ & 10,3 & $7,6 \cdot 11,3$ & 11 & $9,7 \cdot 12,6$ & 10,4 & $9,5-113$ \\
\hline$c$ & 26 & $23-32$ & 26,4 & $21-31$ & 26,9 & $22-30$ & 25,8 & $22.5-28.7$ \\
\hline$V(\%)$ & 72,7 & $67-78$ & & & 73,4 & $72-75$ & & \\
\hline $\begin{array}{l}\text { spicules } \\
\qquad(\mu m)\end{array}$ & & & 27 & $25-30$ & & & 25.3 & $24 \cdot 28$ \\
\hline stylet $(\mu m)$ & 159 & $14-18$ & 14,9 & $14-17$ & 13,5 & $12-14,4$ & 13,05 & $12-138$ \\
\hline
\end{tabular}

Le genre Dotylaphus est signalé pour la première fois en Europe depuis la description de Dotylaphus ruehmi Andrassy, 1958. La seule autre espèce décrite est Dotylaphus lonchites Massey, 1974 associée à Dendroctonus sur différents pins aux Etats-Unis. L'espèce trouvée dans les Landes et le Cher, qui diffère de $D$. ruehmi par la longueur, la position du débouché de la glande dorsale œsophagienne, le nombre de lignes latérales et la forme du stylet, paraît être nouvelle. 
La structure de l'ensemble de cette nématofaune peut être assimilée à celle associée aux Scolytidae (Rühm, 1956), alors que l'un des aspects les plus caractéristiques des cas de mortalité 1 et 2 mentionnés plus haut, réside dans l'absence de xylophages classiques dans la séquence d'installation des ravageurs secondaires. Dans tous les cas en effet, nous n'avons trouvé que des débuts de galeries de longicornes, mais aucune présence significative de Curculionides (Pissodes) ou de Scolyłides (sauf 2 galeries maternelles d'Orthotomicus erosus Woll. sur un pin de $20 \mathrm{~m}$ de haut à Lencouacq !) n'a pu être décelée. Dans les cas de mortalité 4 et 5 , il n'y avait aucun insecte souscortical.

Les premières analyses nématologiques effectuées sur la sciure des galeries et l'insecte lui-même concernent Ips sexdentatus dont les spécimens ont été prélevés dans la région de Lencouacq. Nous retrouvons :

- le Cryptaphelenchus sp. qui est transporté sous les élytres de l'insecte et qui se développe dans les galeries du scolyte ;

- un Allantonematidae avec un stade infestant dans la cavité abdominale de l'insecte ef le stade libre dans la sciure des galeries;

- le Parasitorhabditis sp.

\section{Etudes histopathologiques des arbres infestés}

Des coupes histologiques ont été réalisées dans du matériel (sections de troncs) soumis parallèlement à l'analyse nématologique (fig. 2).

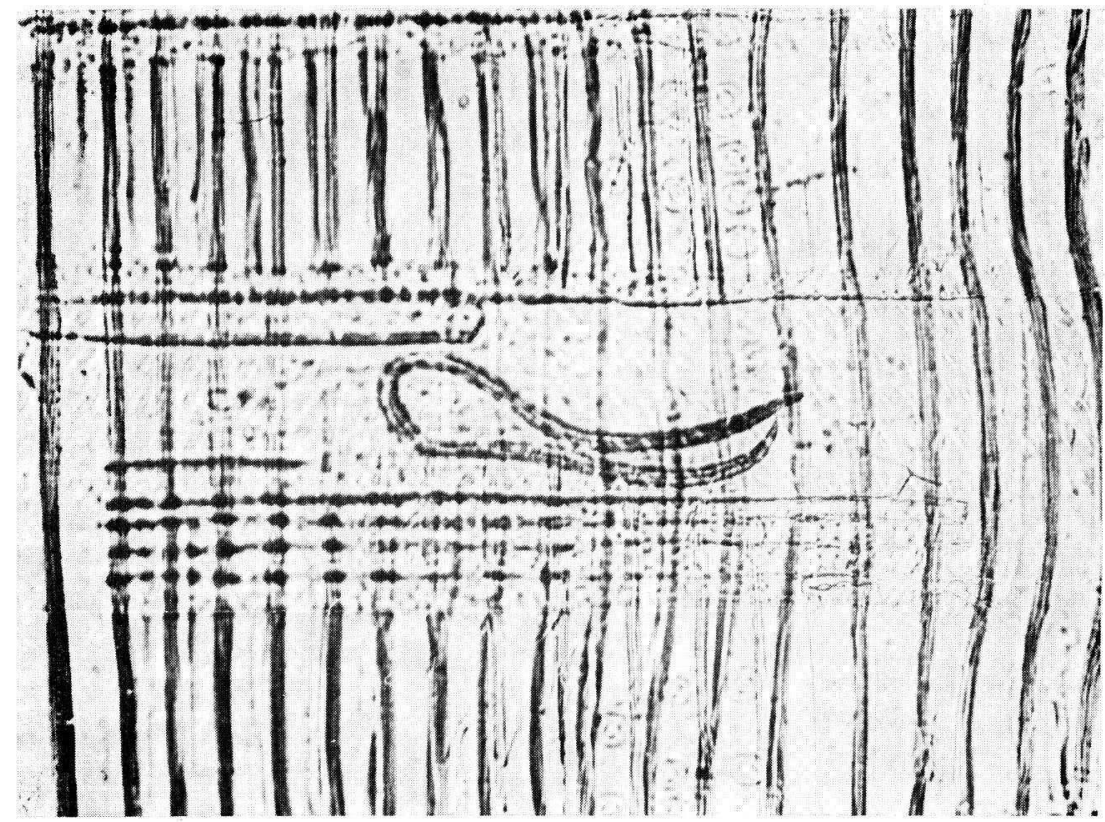

FIG. 2. - Bursaphelenchus n. sp. femelle dans le xylème de Pinus pinaster (Site de Naujac).

A female Bursaphelenchus $n$. sp. in the xylem of Pinus pinaster. 
Après avoir essayé, sans succès, la technique de coloration de Jewell (1958), nous avons utilisé avec de bons résultats la méthode au lactophénol après y avoir apporté quelques modifications en rapport avec la nature du matériel à étudier. Le colorant utilisé est le bleu d'aniline hydrosoluble (anilinblau wasserlöslich - Reagenzien Merck, 1275). Les échantillons de bois, sous forme de petits cubes de $1 \mathrm{~cm}$ de côté, sont plongés dans le mélange lactophénol - bleu d'aniline bouillant pendant $15 \mathrm{mn}$ et demeurent dans le bain jusqu'à refroidissement complet (12 à $24 \mathrm{~h}$ ). Les coupes effectuées sans rinçage, sont déshydratées à l'éthanol, rincées au xylol $(30 \mathrm{mn})$ ef montées au baume du Canada. Les tissus végétaux et les champignons restent incolores alors que les nématodes, colorés en bleu foncé, sont parfaitement repérables. Les premières analyses se rapportent aux pins prélevés sur le site de Naujac. II apparaît que les nématodes (une des nouvelles espèces de Bursaphelenchus) sont répartis dans tous les organes du xylème (canaux résinifères, trachéides) avec une abondance plus grande dans les canaux résinifères.

\section{Conclusion}

Ce travail préliminaire a mis en évidence une nématofaune très particulière dans le tronc de pins maritimes morts ou dépérissants. Aucun nématode n'a, par contre, pu être décelé dans les pins vigoureux ou dans les cas où le mauvais développement est dû à des causes parasitaires identifiées et notamment à l'Armillaire.

Cette nématofaune épigée se révèle très abondante, bien diversifiée et de structure très voisine dans les différents sites échantillonnés. Mais son rôle dans les dépérissements n'est pas encore démontré.

La présence en France de Bursaphelenchus lignicolus, responsable de dégâts sévères sur des Pinus voisins de $P$. pinaster et la sensibilité de cette dernière essence au nématode établie par les chercheurs japonais soulève de nombreux problèmes quant :

- à l'agressivité de la souche française (si toutefois elle s'avère différente de la souche nippone) ;

- à la sensibilité particulière du pin maritime ;

- à l'influence de conditions écologiques des massifs forestiers français concernés, très différentes de celles des zones japonaises, contaminées, sur la nocuité de ce nématode :

- à la nature, l'efficacité et la biologie des vecteurs ;

- au rôle des cryptogames associés à ce nématode.

Ces éléments sont à la base d'une éventuelle stratégie de lutte.

En raison de la dispersion en France de la plupart des nématodes recensés dans cette étude, il nous paraît tout à fait raisonnable de penser que les peuplements forestiers européens de pins maritimes sont susceptibles d'héberger une nématofaune de structure et de composition voisines. 


\section{Summary \\ First data on the nematode-fauna related to « decays» of maritime-pine in western-France}

Unexplained cases of Pinus pinaster decay have caused us to look for nematodes.

A nematological analysis revealed the presence of an important nematofauna in which Bursaphelenchus lignicolus occured: the pathogenicity of this species has already been demonstrated and studied in Japan.

The histological study reveals the presence of these nematodes in resin vessels and wood tracheids.

Our field prospection has brought to light a numerous Sirex population and the presence on standing trees of blue stain fungi.

\section{Références bibliographiques}

BAUJARD P., 1975. Essai d'interprétation de la répartition des groupes trophiques de la nématofaune dans un secteur du massif landais. Mémoire ENITA, Bordeaux, 130 p. (ronéo.).

BEDDING R. A., 1968. Deladenus wilsoni $n$. sp. et D. siricidicola $n$. sp. (Neotylenchidae) entomophagous-mycetophagous nematodes parasitic in siricid woodwasps. Nematologica, 14, 515-525.

BEDDING R. A., 1972. Biology of Deladenus siricidicola (Neotylenchidae) an entomophagous-mycetophagous nematode parasitic in siricid woodwasps. Nematologica, 18, 482-493.

BOUVAREL P., 1960. Note sur la résistance au froid de quelques provenances de Pin maritime R.F.F., no 7, juill. 1960, 495-508.

ILLY G., 1967. Recherches sur l'amélioration génétique du Pin maritime. Ann. Sci. Forest, Tome XXIII, Fasc. 4, 1966, 769-948.

JEWELL F. F., 1968. Stain technique for rapid diagnosis of rust in southern pines. Forest. Sci., 4, 42-44.

KIYOHARA T., TOKUSHIGA, Y., 1971. Inoculation experiments of a nematode Bursaphelenchus sp. into pine trees. J. Jap. For. Soc., 53, 210-218.

LAUMOND C., CARLE P., 1971. Nématodes associés et parasites de Blastophagus destruens Woll. (Col. Scolytidae). Entomophaga, 16, 51-66.

MAMIYA Y., 1972a. Pine wood nematode Bursaphelenchus lignicolus Mamiya and Kiyohara, as a causal agent of pine wilting disease. Rev. Plant. Prot. Res., 5, 46-50.

MAMIYA Y., 1972b. - Reproduction of pine lethal wilting disease by the inoculation of young trees with Bursaphelenchus lignicolus. Jap. J. Nematol., 2, 40-44.

MAMIYA Y., KIYOHARA T., 1972. Description of Bursaphelenchus lignicolus (Nematoda : Aphelenchoididae) from pine wood and histopathology of nematode infested trees. Nematologica, 18, $120-124$.

MASSEY C. L., 1974. Biology and taxonomy of nematodes parasites and associates of bark bettles in the United States. Agriculture Handbook, Forest Service, U.S.D.A., no 446, 233 p.

NICKLE W. R., 1970. A taxonomic review of the genera of the Aphelenchoidea (Fuchs 1937) Thorne 1949 (Nematoda Tylenchida). Journ. Nematol, 2, 375-392.

RUHM W., 1956. Die Nematoden der Ipiden. Parasit. Schriftenreihe, 6, 1-437.

SCOTTO LA MASSESE C., BOULBRIA A., 1974. Essai d'interprétation écologique de la nématofaune de la forêt landaise. XII Symp. Intern. Nématol., Grenade, 1-7 sept.

STEINER G., 1934. Aphelenchoides xylophilus $n$. sp., a nematode associated with blue stain and other fungi in timber. Journal Agric. Res., 48, 949-951. 